\title{
Le temps codé : les calendriers en images (egoyomi)
} au Japon

Encoding Time: Picture Calendars (egoyomi) in Japan

\section{Marianne Simon-Oikawa}

\section{OpenEdition}

\section{Journals}

Édition électronique

URL : http://journals.openedition.org/extremeorient/102

DOI : 10.4000/extremeorient.102

ISSN : 2108-7105

Éditeur

Presses universitaires de Vincennes

Édition imprimée

Date de publication : 30 octobre 2008

Pagination : 145-173

ISBN : 978-2-84292-220-7

ISSN : 0754-5010

Référence électronique

Marianne Simon-Oikawa, "Le temps codé : les calendriers en images (egoyomi) au Japon », ExtrêmeOrient Extrême-Occident [En ligne], 30 | 2008, mis en ligne le 01 octobre 2011, consulté le 03 mai 2019 URL : http://journals.openedition.org/extremeorient/102 ; DOI : 10.4000/extremeorient.102 


\title{
Le temps codé: les calendriers en images (egoyomi) au Japon
}

\author{
Marianne Simon-Oikawa
}

Il est des modes improbables. En 1765, deuxième année de l'ère Meiwa (1764-1771), quelques amateurs fortunés se passionnèrent pour de petits calendriers (koyomi) d'un genre nouveau, qui indiquaient à l'aide d'images (e) la longueur et l'alternance des mois de l'année à venir. Les «calendriers en images» (egoyomi) étaient nés ${ }^{1}$. Pendant deux ans, ils firent fureur. Puis, ils disparurent du devant de la scène ${ }^{2}$. Mais ce qui aurait pu n'être qu'une folie parmi d'autres devait marquer une étape importante dans l'histoire des techniques et des formes artistiques au Japon, favorisant à la fois le développement de l'estampe polychrome ${ }^{3}$ et l'élaboration de nouveaux procédés de cryptage visuels. C'est ce dernier point qui nous retiendra ici.

Circulant dans un milieu fermé constitué de marchands aisés et de samurai amateurs de poésie ${ }^{4}$ qui les échangeaient au cours de réunions organisées spécialement à cet effet (les daishô no kai, littéralement «assemblées de [calendriers indiquant les mois] longs et courts»), les calendriers en images échappaient aux contraintes de la commercialisation et de la diffusion de masse. Cette liberté est à l'origine d'une diversité de moyens rarement égalée. Mais elle explique aussi l'extrême complexité du corpus. La lisibilité et la simplicité sont en effet des notions étrangères aux calendriers en images. Tout chez eux est cryptage, déformation, détournement. Les informations calendaires n'y sont jamais explicites, mais doivent être déduites de l'observation d'éléments cryptés, on serait tenté de dire d' «indices», comme l'alternance d'objets de différentes tailles, ou la présence de caractères traités comme des motifs décoratifs, dissimulés dans les traits constitutifs de la figure (moji-e), ou encore mêlés à un poème ${ }^{5}$.

Le fonctionnement des calendriers en images n'est pas sans rappeler celui d'un code. À la manière d'un langage secret, en effet, ils transmettaient un message (en l'occurrence des informations calendaires) à l'aide d'un ensemble de procédés inaccessibles au plus grand nombre, mais parfaitement 
connus et maîtrisés à l'intérieur du cercle limité dans lequel ils circulaient. Ils nécessitent, pour être compris, la connaissance des règles qu'utilisaient leurs auteurs pour crypter les informations à transmettre, et des compétences que mobilisaient en retour leurs destinataires pour les décrypter.

L'analyse qui suit se propose de dégager quelques-unes de ces règles, à partir d'exemples tirés notamment des collections de la Bibliothèque de la Diète à Tôkyô ${ }^{6}$. En étudiant les principaux types de cryptage à l'œuvre dans ces calendriers en images, séparément ou simultanément, ainsi que les surcodages et glissements génériques qu'opèrent certains d'entre eux, qui empruntent simultanément à plusieurs genres par ailleurs fortement codés, on espère pouvoir ainsi contribuer à une réflexion d'ensemble sur les codes visuels.

\section{Des calendriers pas comme les autres}

Mais, avant de s'intéresser plus avant aux différents moyens inventés par les calendriers en images pour dissimuler dans les figures les indications calendaires, peut-être n'est-il pas inutile de revenir sur ces indications ellesmêmes, dont la complexité nous est devenue, depuis l'adoption du calendrier grégorien au Japon au cours de l'ère Meiji (1868-1912), quelque peu étrangère.

Du VII ${ }^{\mathrm{e}}$ siècle à 1872, le Japon utilisa un calendrier luni-solaire complexe, d'origine chinoise et plusieurs fois modifié au cours de son histoire. Les mois étaient calculés en fonction de la lune. Chaque mois, qui correspondait à une lunaison, comportait, selon les cas et pour obtenir un nombre entier de jours, vingt-neuf (il était alors appelé «mois court», shô no tsuki) ou trente jours («mois long», dai no tsuki). Comme ailleurs, l'année était définie, elle, par le temps que met la Terre pour effectuer un tour complet autour du soleil, soit 365 jours environ. Douze mois de vingt-neuf et trente jours ne totalisant en moyenne qu'un peu plus de 350 jours, il était nécessaire, pour concilier le découpage mensuel avec le découpage annuel, d'ajouter à intervalles réguliers un mois supplémentaire, dit urû no tsuki (閏の月) ${ }^{7}$. Les modalités de cet ajout étaient fixes : il intervenait sept fois au cours d'un cycle de dix-neuf années, aux rangs $1,4,7,10,12,15$ et 18. La place à l'intérieur de l'année et la longueur de ce treizième mois, qui survenait tous les trois ans lors d'années dites «embolistiques», étaient cependant variables.

Les années elles-mêmes étaient désignées en fonction de leur position à l'intérieur de l'ère impériale à laquelle elles appartenaient. L'année 1789 était ainsi la $9^{\mathrm{e}}$ de l'ère Tenmei. Mais leur nom dépendait aussi de leur place à l'intérieur d'un cycle sexagésimal, qui résultait quant à lui de la combinaison de deux systèmes, celui des «douze rameaux terrestres» (jûnishi) et celui des «dix troncs célestes» $(j i k k a n)^{8}$. Chacun des «douze 
rameaux » était désigné par le nom d'un animal et noté à l'aide d'un caractère spécifique: le rat (子), la vache (丑), le tigre (寅), le lapin (卯), le dragon (辰), le serpent (巳), le cheval (午), le bélier (未), le singe (申), le coq (西), le chien (戌) et le sanglier (亥). Cet ensemble de «caractères cycliques» forme ce que l'on appelle aussi communément le «zodiaque chinois». L'année 1789 était ainsi une «année du coq» (toridoshi). Les «dix troncs» correspondaient, eux, aux cinq éléments constitutifs de l'univers selon la pensée corrélative chinoise (bois, feu, terre, métal et eau), dédoublés chacun en «aîné» $(e)$ et "cadet» (to). On disait ainsi : «aîné du bois» (kinoe 甲), «cadet du bois» (kinoto 乙), «aîné du feu» (hinoe 丙), «cadet du feu» (hinoto 丁), «aîné de la terre» (tsuchinoe 戊), «cadet de la terre» (tsuchinoto 己), «aîné du métal» (kanoe 庚), «cadet du métal» (kanoto 辛), «aîné de l'eau» (mizunoe 壬) et «cadet de l'eau» (mizunoto 葵). L'année 1789 était «cadet de la terre» (tsuchinoto). La dénomination complète de l'année 1789 était donc « $9^{\mathrm{e}}$ année de l'ère Tenmei, année du coq, cadet de la terre».

La combinaison de ces multiples systèmes n'allait pas de soi, et l'élaboration d'un calendrier fiable était une affaire de la plus haute importance. Pour éviter toute erreur, la publication et le commerce des calendriers étaient confiés à des éditeurs agréés par le pouvoir (koyomidoiya) et sévèrement contrôlés ${ }^{9}$. Il en existait de plusieurs sortes. Certains donnaient des informations pratiques, comme le moment favorable au repiquage du riz, et fonctionnaient un peu à la manière de nos almanachs. D'autres, destinés aux illettrés, ne contenaient que des images. On vendait aussi des calendriers pliables pour les voyageurs, ou d'autres, longs et minces, destinés à être collés sur les piliers des maisons. Plusieurs particularités régionales étaient aussi autorisées. Les calendriers de Mishima, avec leurs caractères allongés, étaient par exemple très populaires.

Les calendriers en images étaient moins complets. Ils se bornaient à préciser le nombre et l'alternance des mois de l'année, parfois aussi l'animal du zodiaque. Leur réduction à l'essentiel ne les rend pas pour autant plus faciles à lire. Car un calendrier en images est une image cryptée, qui ne peut être comprise qu'au terme d'un processus interprétatif complexe.

Ce processus est encore insuffisamment étudié, pour des raisons qui tiennent à la complexité du corpus des egoyomi lui-même, mais aussi aux difficultés auxquelles se heurtent les spécialistes pour répondre à des questions aussi essentielles que la datation des œuvres, leur attribution, ou encore les raisons exactes de leur succès à partir de $1765^{10}$. De nombreux calendriers sont anonymes, d'autres portent simplement le sceau du groupe d'amateurs qui les commanda, sans que le nom de ses membres soit toujours connu. Certains furent jetés l'année suivant leur distribution; d'autres, collationnés dans des 
albums, l'ont été sans souci d'exhaustivité, parfois sous une forme tronquée. La recherche peine à en donner une idée d'ensemble. Les historiens des calendriers y ont vu essentiellement d'ingénieux supports pour la notation du temps ${ }^{11}$, les historiens de l'art une étape dans le développement de l'estampe polychrome ${ }^{12}$, personne ne s'étant encore attaqué à l'élaboration de critères fonctionnels ou sémiotiques valides pour l'ensemble du corpus. Une observation attentive des egoyomi révèle cependant un faisceau de spécificités notables, instructives dans le cadre d'une réflexion sur les codes visuels.

\section{Compter}

La caractéristique des calendriers en images est qu'ils contiennent un certain nombre d'éléments véhiculant, outre leur signification la plus immédiate, une indication calendaire. Un motif figuratif pourra ainsi servir à désigner une année, ou un mois.

Un animal appartenant aux douze signes du zodiaque a par exemple toutes les chances de constituer une indication calendaire. C'est le cas d'un calendrier pour l'année 1789 représentant un coq, juché sur un tambour (fig. 1). Ce motif traditionnel est attesté dans plusieurs calendriers de notre corpus. Le chant du coq était suivi, dans les demeures de samurai ou dans les châteaux, du battement du tambour indiquant à la population que l'heure de se lever était venue. Le coq sur un tambour constituait ainsi un motif particulièrement propice pour célébrer l'aube d'une nouvelle année du coq. L'oiseau est ici représenté de trois quarts dos, le jabot gonflé et la queue superbement déployée. Majestueux, il annonce une année faste, et c'est naturellement vers lui que se porte d'abord le regard. Un amateur averti sera peut-être tenté de scruter avec attention les détails de son plumage. C'est là en effet que se cache souvent l'indication des mois longs et courts de l'année, les plumes courtes figurant les mois courts (et les longues les mois longs), à moins que les traits servant à dessiner le plumage ne forment eux-mêmes les chiffres des mois. Ces procédés sont parfaitement habituels dans le monde des calendriers en images. Étaient-ils même trop banals ? L'artiste a, semble-t-il, cherché ici à les éviter, et a préféré localiser les indications calendaires dans un motif moins attendu: les feuilles de lierre. Celles-ci sont au nombre de treize et de deux tailles différentes. Les grandes correspondant aux mois longs et les petites aux mois courts, on comprend, en les lisant de haut en bas, que le $1^{\text {er }}$ et le $2^{\mathrm{e}}$ mois sont longs, le $3^{\mathrm{e}}$ court, le $4^{\mathrm{e}}$ long, le $5^{\mathrm{e}}$ court, le $6^{\mathrm{e}}$ long, celui qui suit le $6^{\mathrm{e}}$ est un mois intercalaire (sa couleur moins soutenue, obtenue par un ajout de jaune sur le rouge, indique son statut spécifique), le $7^{\mathrm{e}}$ mois est court, le $8^{\mathrm{e}}$ long, le $9^{\mathrm{e}}$ court, le $10^{\mathrm{e}}$ long, le $11^{\mathrm{e}}$ court et le $12^{\mathrm{e}}$ long. 
Le temps codé: les calendriers en images (egoyomi) au Japon

Figure 1. Calendrier pour 1789 (Tenmei 9, année du coq, cadet de la terre). D'après Okada Yoshirô, Edo no egoyomi, Tôkyô, Taishûkan shoten, 2006, p. 73. 
Réservé à une élite, ce calendrier l'est assurément. Outre son inventivité, son raffinement chromatique le distingue clairement des productions populaires. Son mode de fonctionnement n'a cependant rien d'exceptionnel. Dans les calendriers en images en effet, le nombre des mois ainsi que leur alternance sont souvent indiqués par douze ou treize motifs de tailles ou de couleurs différentes. Ces calendriers étant distribués en mains propres, chacun savait aussi, d'emblée, à quelle année ils correspondaient. Ils n'avaient donc pas pour but d'apprendre à ceux qui les possédaient la configuration de l'année à venir. Tout au plus pouvaient-ils servir d'aide-mémoire aux amateurs oublieux, rappelant que tel mois était court, tel autre long, etc. Ce qui faisait leur intérêt était, bien plus que le contenu de leur message, la manière contournée dont celui-ci était transmis. De fait, l'impunité dont les calendriers en images purent bénéficier tout au long de leur existence, alors même que la production des calendriers commerciaux était sévèrement contrôlée, s'explique peut-être aussi par le fait que les indications calendaires elles-mêmes y étaient finalement secondaires.

Il reste qu'il n'est pas toujours facile de distinguer une scène animalière comme celle-ci d'un calendrier. Sans doute, le nombre de mois n'étant jamais inférieur à douze ni supérieur à treize, il suffisait de chercher dans l'image douze ou treize objets, puis d'observer leur taille respective pour comprendre quel serait leur nombre exact cette année-là, ainsi que leur alternance. Mais encore fallait-il, pour avoir l'idée de ce décompte, avoir d'abord identifié l'image comme un calendrier. Or, pour éviter l'artifice d'une image trop explicite, surexposant les indices et par là même sans intérêt, les artistes ont cherché à rendre l'alternance la plus naturelle possible, la localisant dans des motifs eux-mêmes complexes, comme les ramifications végétales, les plumages ou les pelages d'animaux divers.

La connaissance du code et l'habitude du regard étaient des conditions essentielles à l'interprétation. Or elles sont aujourd'hui perdues. Le chercheur doit, pour les déchiffrer, mobiliser des connaissances oubliées, retrouver les réflexes des premiers amateurs. Il doit se reporter, par exemple, à des tables chronologiques indiquant, pour chaque année, le nombre des mois, leur longueur et leur alternance. Dans le cas d'un calendrier représentant un coq, il supposera d'abord que l'animal désigne une année du coq et cherchera, parmi les années du coq, celle dont la configuration correspond aux informations identifiées dans l'image. Si aucune année n'est identique aux indices observés, les critères de recherche devront être modifiés. Peut-être le calendrier ne correspond-il pas, en réalité, à une année du coq? Peut-être un détail non repéré indiquait-il l'existence d'un mois intercalaire? La présence de petites bandes de papier verticales collées sur plusieurs calendriers des 
albums de la Bibliothèque de la Diète et mentionnant l'année à laquelle ils correspondent, est là pour témoigner du labeur de ceux qui, bien longtemps après leur publication, s'attachèrent à les décrypter.

Les hommes du temps semblent s'être eux-mêmes parfois perdus dans la complexité de leurs propres codages, ou du moins avoir craint que leurs cryptages, trop subtils, ne soient pas découverts par ceux auxquels ils étaient destinés. Il existe des calendriers erronés ${ }^{13}$, d'autres dans lesquels les artistes ont clairement daté leur image. Dans la partie inférieure droite de notre calendrier de 1789, par exemple, l'artiste a ainsi indiqué très lisiblement, à la place de son nom, «Tenmei 9». Le sceau vermillon apposé sur l'estampe à la façon d'un sceau d'artiste indique, lui: 己西大小 (tsuchinoto tori daishô, «calendrier de l'année du coq, cadet de la terre»). Sans doute est-ce là la preuve que ces calendriers étaient avant tout ludiques, destinés à exercer la sagacité de leur propriétaire dans la découverte d'une énigme, d'autant plus attirante que sa solution était donnée d'avance. Mais on peut voir aussi dans ces indications l'expression de la conscience que les artistes eux-mêmes avaient des limites des codes qu'ils employaient. Trop finement crypté, notre calendrier de 1789 courait le risque de devenir incompréhensible.

De nombreux calendriers reprennent le schéma fondamental que ce coq de 1789 nous a permis d'observer, associant animal du zodiaque et objets de différentes tailles pour figurer les mois. À titre de variation, ils peuvent aussi représenter, au lieu de l'animal proprement dit, un personnage ou un objet qui lui est habituellement associé. Un Daikoku juché sur deux balles de riz vaudra ainsi pour l'année du rat, animal qui se nourrit de graminées et qui figure parmi les attributs traditionnels de cette divinité protectrice des cultures.

\section{Décorer}

C'est parfois dans l'ornementation de certains objets que se cachent les indications calendaires. Elles prennent alors la forme de chiffres, traités comme des éléments décoratifs. L'un des supports où ces chiffres se trouvent le plus volontiers (mais non exclusivement) est le vêtement des personnages. Les kimono réellement portés à l'époque étaient souvent décorés de caractères d'écriture (poèmes, formules de politesse, etc). Il était d'usage aussi, dans les illustrations des romans populaires, de signaler les personnages représentés par un des caractères de leur nom, enfermé dans un cercle blanc et situé à un endroit bien visible de leurs vêtements. On comprend que des chiffres aient pu remplacer sans choquer les signes graphiques habituels.

Dans un autre calendrier pour l'année 1789, on voit ainsi deux enfants vêtus et coiffés à la chinoise, en train de jouer avec un cerf-volant (fig. 2). Les 
Figure 2. Calendrier pour 1789 (Tenmei 9, année du coq, cadet de la terre). Tiré d'Okada, op. cit., p. 43.

indications calendaires sont dissimulées à la fois dans le motif du jouet, un coq, et dans le kimono de l'enfant qui tire le cerf-volant. On lit, dans les cercles qui décorent le tissu, les caractères qui correspondent aux mois courts: 三 (3, épaule gauche), 五 (5, épaule droite), 壬六 (urû 6, taille, l'année comportant un mois intercalaire, celui qui suit le $\left.6^{\mathrm{e}}\right)$, 七 (7, bas de la manche gauche), 九 (9, jambe gauche) et 十一 (11, bas du dos) ${ }^{14}$. Les mois longs ne sont pas mentionnés dans l'image, mais peuvent en être aisément déduits: sont longs les mois qui ne sont pas courts... Comment toutefois savoir que les mois mentionnés sont précisément les mois courts? Souvent, un élément dans l'image vient le préciser: le motif où ils sont mentionnés est plus court (petit, ou mince) que les autres, à moins que le caractère 小 (petit) ne soit lui aussi indiqué. Rien de tel ici. Les deux enfants ont sensiblement la même taille. Le recours aux tables chronologiques est ici le principal moyen de dater l'image.

Dans ces calendriers, les chiffres décorent la figure, mais leur absence n'empêcherait en rien l'image d'exister. Tout au plus lui enlèverait-elle sa fonction calendaire. Si un éditeur jugeait une image particulièrement réussie, 
il pouvait d'ailleurs racheter les planches ayant servi à sa réalisation et demander ensuite à un graveur d'effacer toute trace calendaire, avant d'imprimer une nouvelle estampe et de la mettre en vente. Les signes indiquant l'alternance des mois courts et des mois longs disparaissaient, éventuellement remplacés par d'autres motifs décoratifs divers. Périmé, le calendrier n'était plus qu'une image comme une autre et pouvait être diffusé dans le circuit habituel. Certains calendriers en images passèrent ainsi dans le circuit commercial, après la fin de l'année pour laquelle ils avaient été imprimés, cette fois amputés de toute indication calendaire. De nombreux calendriers en images de Harunobu connurent ainsi deux versions: l'une proprement calendaire, destinée à une distribution privée et réalisée à grands frais, et une autre moins luxueuse, partiellement regravée, d'où les chiffres ont été effacés ${ }^{15}$.

\section{Écrire le temps : le recours au moji-e}

Tel n'est pas le cas des calendriers qui recourent au moji-e. Bien antérieur aux egoyomi eux-mêmes, le moji-e est l'un de leurs procédés de prédilection. Héritier de pratiques ludiques attestées depuis l'époque de Heian (794-1185), il consiste à utiliser des caractères d'écriture (moji) pour former des figures (e). Il prend le plus souvent la forme d'un personnage dont le vêtement est constitué des caractères indiquant son métier: «ha-na-u-ri» par exemple, pour un marchand de fleurs (hana-uri). Le moji-e est en lui-même un procédé de cryptage ${ }^{16}$. Il consiste, en effet, à dissimuler, à l'intérieur d'une figure, des traits d'écriture savamment déformés, dont la combinaison constitue un mot. Le spectateur doit, pour le comprendre, d'abord repérer les caractères qui s'y trouvent utilisés, puis reconstituer leur combinaison. Dans les calendriers en images, les caractères choisis pour constituer le moji-e sont simplement les chiffres des mois de l'année.

On voit ainsi, dans un des calendriers de la Bibliothèque de la Diète pour l'année 1859 (Ansei 6, année du bélier, cadet de la terre), un marchand ambulant, commercialisant un remède fameux appelé gama no abura (littéralement «huile de crapaud») (pl. 24, p. 109). Un moine du Mont Tsukuba ayant appliqué cette crème sur la blessure d'un guerrier dont la tête ressemblait à celle d'un batracien, le médicament connut un succès immédiat et fut commercialisé sous le nom d' "huile de crapaud». Ces produits étaient vendus en ville dans des boutiques, mais aussi par des marchands ambulants, proches des saltimbanques, qui attiraient le public en exécutant dans la rue des numéros d'acrobatie. Souvent accompagnés d'un acolyte et munis d'un sabre, ils dansaient sur de petits plateaux empilés les uns sur les autres. 
Le marchand d'«huile de crapaud» est ici représenté dans une pose périlleuse, tandis que son assistant le regarde, accroupi à côté de lui. Le vêtement de l'acrobate et les trois plateaux qui soutiennent son pied droit sont obtenus à l'aide des caractères correspondant aux mois longs. Il faut deviner les caractères 十二 (12) dans la manche droite du vêtement, 十一 (11) dans la manche gauche, 九 (9) dans le bas de la manche gauche (ses lignes fluides, audessus du poignet et dans la manche, le rendent presque indétectable), 六 (6) dans le sabre et la jambe droite, 正 ( $1^{\text {er }}$ mois) dans le bas du vêtement et 三 (3) dans les plateaux empilés. Les mois courts forment, eux, les vêtements de l'assistant: 七 (7, cheveux), 二 (2, manche gauche), 五 (5, dos), 八 ( 8 , manche droite), 四 (4, hanches) et 十 (10, sabre).

Ce calendrier est associé à d'autres éléments dessinés ou écrits. Les figures, on l'a sans doute remarqué, ne sont pas entièrement réalisées à l'aide de caractères. Conformément à la forme canonique du genre, la tête, les mains et les pieds des personnages sont dessinés. Due à un disciple de Katsushika Hokusai (1760-1849), Yashima Gakutei (1786-1868) - qui signe ici Gakutei Ryôsa, nom qu'il prit à la fin de sa vie -, la figure est accompagnée d'un poème de Kanagaki Robun ${ }^{17}$, qui dit: Jômise no kazari ni gama no tachikatana dai Dainoshin yuzuriha ni koso («Devant une boutique, le grand Dainoshin avec son sabre fait de la publicité, Daphniphyllum macropodum»). Dainoshin est un simple prénom de convention. Plus intéressant pour nous est le Daphniphyllum macropodum (yuzuriha). Cette plante, dont les nouvelles feuilles poussent par-dessus celles de l'année précédente, est l'image au Japon du remplacement d'une génération par une autre, qui lui cède pour ainsi dire sa place (yuzuru). Elle constitue un motif faste, particulièrement bienvenu au Nouvel An, et son nom, bien plus évocateur en japonais qu'en français où cette plante ne semble pas avoir de nom vulgaire, véhicule une indication temporelle (le début de l'année).

\section{Des poèmes calendriers}

Les poèmes qui accompagnent les images méritent parfois une attention particulière. Il arrive en effet qu'ils doivent eux-mêmes se lire comme des calendriers. Éventuellement associés à une figure avec laquelle ils fonctionnent alors de manière complémentaire, ils recèlent en eux-mêmes l'indication des mois courts et des mois longs. Certains calendriers associent, à l'intérieur d'un même espace, plusieurs types de cryptage et se trouvent ainsi doublement, voire triplement codés.

C'est le cas par exemple d'un calendrier de 1866 (Keiô 2, année du tigre, aîné du feu), qui représente une femme marchant sous un saule (pl. 25, p. 109). 
Très raffiné, avec ses dégradés de couleur dans le ciel et le vêtement du personnage, ce calendrier donne d'abord une impression de douceur. Qui pourrait, à seulement regarder la figure, se douter qu'il s'agit d'une année du tigre (tora) ? L'animal du zodiaque n'est pas représenté. Est-ce à dire qu'il soit tout à fait absent de l'image? Il y est en réalité mentionné pour ainsi dire en toutes lettres: les caractères と («to») et $ら$ («ra») servent à figurer, et très lisiblement, le tronc de l'arbre, dans la partie supérieure droite du calendrier. L'alternance des mois, quant à elle, est repérable dans le texte du poème. Avant même de le lire, à simplement le regarder, on s'aperçoit que celui-ci contient les chiffres 六 (6), 二 (2), 四 (4), 五 (5) et 九 (9), sous une forme plus ou moins cursive. Ces chiffres ont d'abord vocation à être lus. Comme en français où, de façon ludique, on écrit parfois le mot «cassette» à l'aide de la lettre K et du chiffre 7 , à la manière de rébus de lettres, la forme sonore des chiffres permet ici de constituer le poème. Celui-ci se lit: Harusame ya mume wa nikoniko shigoku yoshi, ce qui signifie simplement: «Pluie de printemps, le prunier sourit, c'est magnifique». Plus que la densité poétique de ces vers que la postérité n'a pas retenus, ce qui est en jeu ici est l'ingéniosité avec laquelle ils ont été transcrits. Car leur auteur a réussi à y intégrer, dans une combinaison sonore, sans doute artificielle mais qui au moins fait sens, les cinq chiffres des mois courts de l'année: 6 (lu «mu», de mume), 2 («ni», de nikoniko), 4 («shi»), 5 («go») et 9 («ku») formant le mot shigoku. Ce n'est pas tout. La figure elle-même sert de support à une partie des indications calendaires. Le vêtement de la jeune femme est en effet constitué à l'aide des caractères correspondant aux mois longs. On lit en effet dans ses cheveux le caractère dai (大, «long») et 三 $(3$, on remarque trois traits fins figurant les ornements de

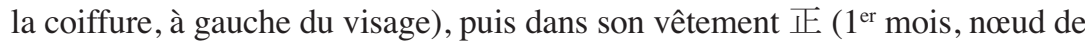
la ceinture), 七 ( 7 , bas du kimono $)$, 十 (10, jambe gauche, à la frontière du kimono et de la ceinture), 十一 (11, épaule gauche), 十二 $(12$, dos $)$ et 八 (8, pieds).

La répartition entre le texte et l'image est ici particulièrement complexe. Loin de se limiter aux deux parties d'un calendrier (les mois courts dans le poème, les mois longs dans la figure), elle ne correspond pas non plus à une simple division entre deux médias différents, le texte à gauche, la figure à droite (auxquels il conviendrait d'ailleurs d'ajouter les sceaux). Elle donne plutôt à voir deux types de relations entre le texte et l'image. Dans le texte, les caractères qui notent les chiffres sont dotés simultanément de deux valeurs: ils fonctionnent comme indicateurs numériques dans un calendrier (comme chiffres, ils se signalent au regard avant même d'être lus) et comme sons à l'intérieur du poème. Dans la figure aussi leur valeur est double: ils constituent des indicateurs numériques, mais aussi des traits permettant de constituer une 
figure (moji-e). Des calendriers de ce type ne sont pas rares. Les amateurs, loin d'être déconcertés par de telles combinaisons, devaient particulièrement apprécier ces superpositions graphiques, scripturaires et sonores.

\section{Écrire des mots avec des chiffres}

Les chiffres, nous venons de le voir, peuvent être utilisés pour noter des sons. Ils peuvent aussi servir à écrire des mots. Ils rejoignent ici le moji-e, mais par un détour curieux. Car si les moji-e classiques font des dessins avec des mots, et nos acrobates calendaires des dessins avec des chiffres, les images qui nous intéresseront ici font des mots avec des chiffres. Comment? En remplaçant habilement les traits constitutifs de certains sinogrammes, par les chiffres correspondant aux mois de l'année. La dissimulation est habile, et l'exercice du regard, peut-être plus encore qu'ailleurs, essentiel au décryptage de l'image.

L'un des sinogrammes privilégiés des calendriers de ce type est le caractère 壽 (kotobuki), qui signifie «longévité» (pl. 26, p. 110). Fréquent dans les images de bon augure, on le trouvait, dans la vie quotidienne, sur toutes sortes d'objets qu'il servait à décorer, en même temps qu'il leur communiquait sa puissance propitiatoire. Les moji-e eux-mêmes en font grand usage. Dans les calendriers en images, kotobuki apparaît volontiers sur des objets utilisés au Nouvel an, comme des coupes de saké. Dans ce calendrier de l'année 1857 (Ansei 4, année du serpent, cadet du feu), la coupe est décorée du caractère kotobuki, lui-même constitué de chiffres qui se lisent, de haut en bas et de gauche à droite: 十二 (12), 五 (5), 六 (6), 八 (8, deux traits disposés de part et d'autre de l'ensemble que forment le 5 et le 6 ), 十一 (11), 九 (9) et 二 (2, à l'intérieur du 9). Le poème contient l'autre moitié des indications calendaires: 七 (7), 五 (5), 三 (3), 正 (1 ${ }^{\mathrm{er}}$ mois), 十 (10) et 四 (4). Rien dans ce calendrier n'indique l'année pour laquelle il fut publié, mais la comparaison des deux séries de chiffres avec les tables chronologiques montre qu'il s'agit de 1857. La coupe contient les mois longs, le poème les mois courts. La réalisation de ce calendrier est luxueuse: aux dégradés (dans la partie supérieure de l'image et dans la coupe elle-même), s'ajoute l'or des caractères et de tous les traits du dessin. Comme dans le poème placé audessus de la belle promeneuse de 1866, les chiffres sont utilisés ici pour noter des mots, mais dans une perspective ludique plus explicite encore. Saturé de jeux de mots, le poème dit en effet: Shichi (7) go (5) san (3) yufute masayume mitari toso no yoi ( «De beaux atours pour la fête des enfants, un rêve prémonitoire, ivresse du Nouvel An»). L'allusion à la fête des enfants constitue sans doute simplement ici une expression faste, appropriée aux célébrations du Nouvel An, même si elle a lieu à une autre date ${ }^{18}$. 
Dans l'expression masayume, qui désigne un rêve (yume) qui devient «vrai» en se concrétisant dans la réalité, le caractère masa (正) fonctionne à la fois comme adjectif («vrai»), et comme indicateur calendaire («1 er $^{\text {er }}$ mis $\left.»\right)$. Le cas de toso no yoi - qui joue sur le double sens de yoi («bon»良い et «ivre» 酔い) - est plus complexe. Le premier caractère du mot toso (屠蘇, saké du Nouvel an) a en effet été remplacé par le chiffre 10 (十, to), et la première syllabe de yoi (yo, 良 ou 酔) par le chiffre 4 (四). Cette substitution de chiffres aux caractères attendus rejoint le procédé que nous avons identifié dans le calendrier de 1866. Mais elle prend ici une valeur particulière puisque, dans la coupe aussi, le caractère kotobuki est remplacé par des chiffres.

Un calendrier de 1854 (Ka.ei 7, année du tigre, aîné du bois) cumule presque tous les procédés rencontrés jusqu'ici (pl. 27, p. 110). L'année, imprimée dans la partie droite de l'image (Ka.ei 7), y est aussi indiquée par un jouet en forme de tigre. Ce tigre lui-même est un moji-e obtenu à l'aide des caractères 正 (1 $1^{\mathrm{er}}$ mois), 三 (3), 五 (5), 壬七 (urû 7), 九 (9) et 十一 (11), déformés de manière à se fondre dans le contour de l'animal. Le poème qui accompagne la figure principale complète le calendrier. Son premier caractère 大 (dai), qui fait partie du mot taihei, indique aussi qu'il contient la mention des mois longs. Ceux-ci, de fait, doivent être devinés dans le caractère «féliciter» (kotohogu 壽), lui-même obtenu à l'aide de chiffres (de haut en bas et de gauche à droite): 十二 (12), 四 (4), 六 (6), 二 (2), 七 (7), 八 (8) et 十 (10). Le poème se lit: Taihei no kotohogu miyoya tora no haru («À notre heureuse époque de grande paix, printemps du tigre»).

La surenchère est sans fin. Les procédés de cryptage peuvent se combiner à l'envi, surcodant des images qui pourraient, pour des néophytes, paraître univoques. La surcharge dont témoigne ce calendrier peut sans doute à elle seule éveiller les soupçons du lecteur-spectateur, mais elle ne donne pas pour autant les clés de son déchiffrement. Les procédés de cryptage peuvent aussi connaître plusieurs degrés et même, faisant intervenir un nouveau type de codage, jouer avec la conscience que le spectateur a de genres connexes auxquels ils empruntent certains procédés.

\section{Du dessin au moji-e : les calendriers en images aux frontières des genres}

Il est, par exemple, intéressant d'observer comment un procédé comme le moji-e peut être plus ou moins activé dans des images calendaires, et même s'y trouver transformé. La comparaison entre plusieurs calendriers prenant pour sujet les mêmes motifs est à cet égard particulièrement éclairante. Le calendrier au coq de 1789 (fig. 1), par exemple, ne contenait aucun moji- 
$e$. Le cryptage reposait tout entier sur le dessin, les motifs représentés et leur nombre. Un autre calendrier, lui aussi élaboré pour l'année du coq, mais publié soixante ans plus tard, en 1849 (Ka.ei 2, année du coq, cadet de la terre), utilise au contraire toutes les ressources du moji-e (fig. 3). Le plumage de l'oiseau abrite les mois courts: le caractère 小 («petit») est dissimulé dans son jabot, 二 (2) dans l'œil et le bec, 四 (4) dans la crête, 六 (6) dans la patte, 八 (8) dans la queue et 十一 (11) dans le ventre. Le mois intercalaire est celui qui suit le $4^{\mathrm{e}}$ (urû 壬 sur le dos et 4 四 dans l'aile). Le tambour contient, lui, les mois longs (son socle n'est autre que le caractère 大 «grand»). On doit deviner dans les veines du bois, de haut en bas et de gauche à droite, les caractères 九 (9), 正 ( $1^{\mathrm{er}}$ mois), 十二 (12), 五 (5), 七 (7), 三 (3) et 十 (10). Ce moji-e est en outre accompagné d'un poème. Sa veine comique témoigne de son souci de divertir par son ingéniosité verbale. Il se lit: Dondon to sakaeru

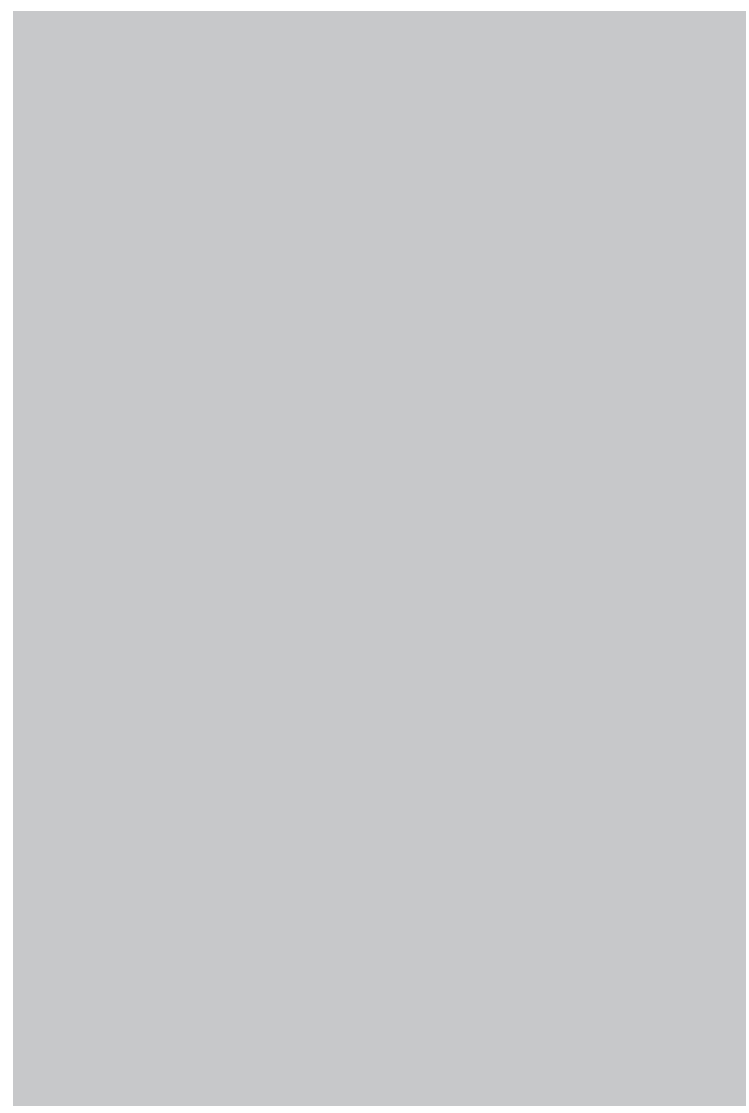

Figure 3. Calendrier de 1849 (Ka.ei 2, année du coq, cadet de la terre). Egoyomi, Bibliothèque de la Diète. 
miyo no tokitsukuru ô kekkô to toshi wo toridoshi, et consiste en une suite de jeux de mots qui décourage la traduction. Dondon signifie «de plus en plus» en même temps qu'il correspond au bruit du tambour, kekkô to peut se traduire par «convenable» autant que par «cocorico», et toshi wo toridoshi associe l'expression toshi wo toru («vieillir») à toridoshi («année du coq»). Le battement du tambour et le cri du coq se superposent pour annoncer (tokitsukuru) une année prospère (sakaeru).

Faut-il voir simplement, entre ce calendrier et celui de 1789, deux interprétations d'un même sujet à l'aide de deux procédés différents? Le coq sur un tambour est, on l'a dit, un motif fréquent dans les calendriers en images. Dans le cas qui nous occupe, une troisième étape semble cependant avoir pu faciliter le passage de notre premier calendrier au second. Due à Hokusai et figurant dans son Ono ga Bakamura muda jie-zukushi ${ }^{19}$ (1810-1812), elle représente un coq. Dénuée de toute fonction calendaire, elle recourt cependant à la fois au dessin et au moji-e (fig. 4). Rappelons-en rapidement le contexte: dans les années 1810, Hokusai publia plusieurs manuels de dessin qui mettaient à la disposition d'élèves et d'artisans un ensemble de modèles à copier. Ono ga Bakamura muda jie-zukushi est de ceux-là. Destiné plus particulièrement aux poètes de kyôka, qui se plaisaient au cours de leurs réunions, à rivaliser d'ingéniosité non seulement en composant des poèmes, mais encore en dessinant de petites figures, il se donne pour mission de leur enseigner comment tracer en quelques instants de petits motifs simples ${ }^{20}$. Les calendriers en images eux-mêmes, on s'en souvient, naquirent dans des cercles d'amateurs souvent férus de kyôka.

L'ouvrage se veut avant tout utile et sa structure est éminemment pédagogique. Chaque page contient ainsi une image, accompagnée d'un commentaire qui en explique la construction. Elle est divisée en plusieurs registres compartimentés. Le plus grand, en bas à droite, contient la figure principale (ici le coq), éventuellement accompagnée d'une figure secondaire (les pous-

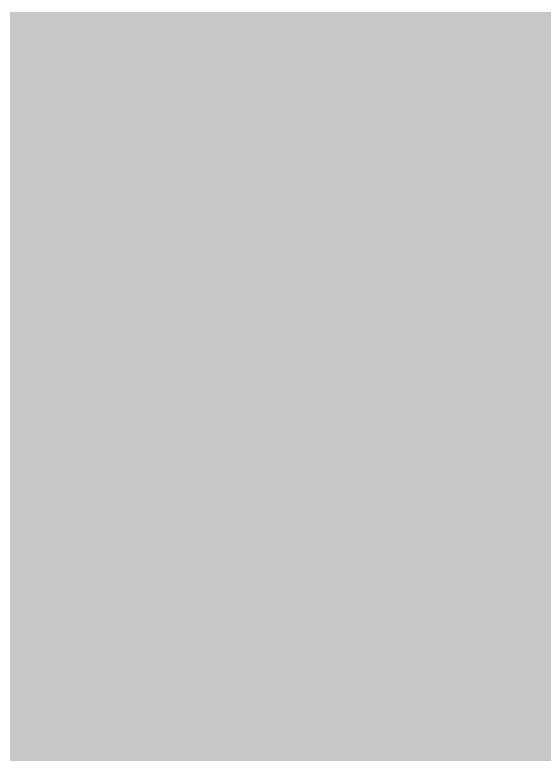

Figure 4. Hokusai, Ono ga Bakamura muda jie-zukushi (1810-1812). D’après Nagata Seiji (dir.), Hokusai no e-dehon, vol. 1, Iwasaki bijutsusha, 1986, p. 42. 
sins). Il contient aussi un court texte qui commente, sous la forme d'un kyôka, la construction de la figure secondaire. Le commentaire de la figure principale, lui aussi un kyôka, se trouve dans le compartiment vertical placé sur la gauche. Le compartiment supérieur comprend, à droite, les éléments constitutifs de la figure principale, soigneusement séparés les uns des autres et numérotés dans l'ordre où ils doivent être tracés, et à gauche, les éléments, numérotés ou non, de la figure secondaire. Cette répartition, complexe au premier abord, permet en réalité de suivre pas à pas la réalisation de la figure, sans rien y oublier d'important. Dans l'exemple qui nous occupe, le coq est représenté de profil, tourné vers la gauche et juché sur un tonneau. Il est constitué de traits simples, mais aussi de caractères d'écriture: le caractère 小 («petit») sert à dessiner le bec, les chiffres 八 (8) et 六 (6) les pattes.

Plusieurs motifs d'Ono ga Bakamura muda jie-tsukushi sont attestés dans des recueils antérieurs dus à des poètes de kyôka. Il n'est pas impossible que Hokusai se soit inspiré, pour élaborer sa figure, de coqs comme celui de notre calendrier de 1789, même si rien n'indique qu'il se soit fondé sur cette image en particulier. Il est encore plus vraisemblable que le coq d'Ono ga Bakamura muda jie-zukushi ait inspiré le calendrier de 1849, dans lequel la pose du coq, le tonneau et jusqu'à certains détails comme le traitement et la place du chiffre 6 sont identiques. Inspiration n'implique toutefois pas copie. Le coq de Hokusai ne constituait pas un moji-e complet, ni un calendrier. Il recourait à de nombreux éléments autres que des caractères d'écriture et n'avait aucune vocation calendaire. Celui de 1849 , tout entier moji-e, calendaire et comique, en constitue davantage un prolongement et une métamorphose.

\section{Du moji-e classique au moji-e calendaire}

À l'intérieur du moji-e lui-même, la comparaison entre les formes classiques du procédé et celles qu'il prend dans les calendriers en images fait elle aussi apparaître les continuités et les transformations survenues au cours du passage de l'un à l'autre. Le moji-e, on l'a dit, est un procédé ancien. L'acrobate et son assistant, par exemple, ne sont pas des créations originales de notre calendrier de 1859. Ils reprennent un type de représentation attestée dès les premiers recueils de moji-e qui nous soient parvenus, le Moji-e tsukushi («Recueil de moji-e», 1685) et le Shin Moji-e tsukushi («Nouveau recueil de moji-e», 1751-1764).

Le Moji-e tsukushi ne représente qu'un seul personnage, l'acrobate, en pleine action (fig. 5). L'omission de l'assistant ne doit pas surprendre. Elle est congruente avec le minimalisme du recueil tout entier qui se limite dans la quasi-totalité des cas à un personnage par page, en gros plan ${ }^{21}$. Le moji-e 
Figure 5. «Marchand de hangontan», Moji-e tsukushi, $1836 \quad\left(1^{\mathrm{re}}\right.$ édition 1685). Coll. de l'auteur.

est localisé dans le vêtement du personnage, dessiné à l'aide des caractères qui se lisent Toyama-hangontan («Hangontan de Toyama») : と (to) sert à former le col du kimono, 山 (yama) la manche gauche, «ha» (signe syllabique cursif ou kana issu du kanji 者) la manche droite, $ん(n)$ la ligne du torse, ごん (gon) la jambe droite, «ta» (kana issu du kanji 多) et $ん$ (n) la jambe gauche. Le texte placé dans la partie inférieure gauche de l'image reprend les mêmes caractères sous une forme linéaire et parfaitement lisible (du moins pour les contemporains), et permet au lecteur de s'assurer qu'il a correctement identifié tous les caractères présents dans le vêtement du personnage.

Le Shin Moji-e tsukushi reprend la même scène, mais sous une forme plus développée: l'acrobate est représenté en compagnie de son acolyte, la décoration du kimono et celle des plateaux est plus poussée, et on voit à l'arrière-plan de la scène un début de décor (fig. 6). Le titre de l'image est indiqué sur le bord droit: Matsuiya hangontan to ifu moji-e («Moji-e dit 
hangontan de la maison Matsui»). Le moji-e proprement dit est localisé dans le vêtement de l'acrobate. Il se lit, de fait: Matsuiya hangontan. On y repère les caractères 松 (matsu) au milieu de la manche droite, 井 $(i)$ au niveau de l'épaule droite, や $(y a)$ dans l'épaule gauche, «ha» (kana issu du kanji 者) dans la manche droite, $ん(\ll \mathrm{n} »)$ dans la jambe droite, こん («kon») dans la manche gauche, «ta» (kana issu du kanji 多) et $ん$ («n») dans la jambe gauche. L'assistant ne contient, lui, aucun caractère. Les paroles que lui adresse l'acrobate sont mentionnées dans le texte de la partie supérieure de l'image: «Eh, serviteur, tu as compris? Viens frapper! Tout est dans le mouvement des reins. Un bon médicament contre les vers intestinaux, le hangontan de Toyama, dans le pays d'Etchû ${ }^{22}$ !» (Yai yakko kokoroheta ka utte koi koshi no hineri ga daiji ja mushi ichidô ni yoi Etchû Toyama hangontan.) L'image du Shin Moji-e tsukushi est plus détaillée que celle du Moji-e tsukushi, mais les deux acrobates en moji-e, qui partagent la même identité structurelle, témoignent de la permanence du genre et de ses traits distinctifs : un personnage dont le vêtement est dessiné à l'aide des caractères qui indiquent son nom.

Figure 6. «Marchand de hangontan», Shin Moji-e tsukushi, 1751-1764. Bibliothèque centrale de la ville de Tôkyô. 
À la différence de nos deux moji-e précédents, le calendrier a tiré parti de la présence des deux figures (pl. 24, p. 109). Non que le grand nombre de chiffres eût rendu impossible leur présence dans les vêtements d'un seul personnage ( $d$ 'autres calendriers font tenir les douze mois dans un très petit espace), mais plus vraisemblablement parce que ce tandem correspondait idéalement à la binarité des indications calendaires elles-mêmes, faites de l'alternance entre des mois courts et des mois longs. Les calendriers pour illettrés symbolisaient volontiers les mois longs par un sabre long et les mois courts par un sabre court. Dans le Shin Moji-e tsukushi, l'assistant, dont le statut est inférieur et qui est aussi représenté accroupi, un sabre court dans la main droite, contient les mois courts. L'acrobate, personnage principal de la scène, figuré debout sur les plateaux et armé d'un sabre long, prend en charge les mois longs.

La mise en regard de ces trois images montre assez le profit que les calendriers en images pouvaient tirer du moji-e: le procédé, qui assignait luimême deux valeurs distinctes à certains tracés, à la fois dessins et caractères d'écriture, était particulièrement propice à l'élaboration de nouveaux codes visuels. Mais cet emprunt ne put se faire sans un certain nombre d'aménagements à la fois formels et thématiques, destinés à le rendre compatible avec les exigences des calendriers en images, et en premier lieu l'utilisation de chiffres et le recours à des motifs non humains. Ces procédés aboutirent à la constitution, à l'intérieur du genre des calendriers lui-même, d'une nouvelle catégorie d'images doublement codées, à la fois comme moji$e$ et comme calendriers.

\section{Les métamorphoses d'un arbre aux feuilles d'or}

Le moji-e n'est pas le seul procédé à se voir ainsi revisité dans les calendriers en images. Les «arbres aux feuilles d'or» (kane no naru ki), groupe par ailleurs très proche des moji-e mais constitué en genre autonome, comme l'indique le fait même qu'il soit doté d'un nom spécifique, présentent eux aussi quelques transformations intéressantes.

Les images qui appartiennent à cette catégorie représentent immanquablement un arbre dont les feuilles sont constituées de pièces d'or et dont le tronc et les branches sont dessinés à l'aide de formules invitant au travail et à la vertu. Toutes se terminent par le son $k i$, lecture japonaise du mot «arbre», mais le plus souvent utilisé comme simple terminaison adjectivale: asaoki («matinal»), shinbô zuyoki («persévérant»), shôjiki («honnête»), etc. Une estampe d'Utagawa Yoshinobu publiée entre 1848 et 1860 présente un exemple canonique du genre (fig. 7). On y voit les dieux Ebisu, une daurade dans une 


\section{Marianne Simon-Oikawa}

main, une canne à pêche dans l'autre, et Daikoku, juché sur deux balles de riz, un gros sac sur le dos, assis de chaque côté d'un arbre tout entier tracé à l'aide de caractères d'écriture et dont les feuilles sont constituées de pièces d'or. Certaines sont même tombées aux pieds des deux divinités. On aperçoit aussi entre les deux personnages une branche de corail, accessoire fréquent des images fastes en raison de son nom, sango, qui par homophonie peut faire penser à une nombreuse descendance (littéralement «après la naissance»). Le texte placé dans le registre supérieur de l'image dit: Yudan naku kasegu kono mi no asemizu wa kane no naru ki no koyashi nakaran («Travailler sans relâche pour gagner sa vie. La sueur nourrit l'arbre aux feuilles d'or $\left.{ }^{23} »\right)$. Le rêve d'un arbre produisant de lui-même la richesse n'exprimait rien d'autre que le désir de voir ses efforts récompensés. On lit, dans la partie droite de l'arbre, de haut en bas: asaoki («matinal»), isagi no yoki («résolu»), yôjô yoki («en bonne santé»), shinbô zuyoki («patient») et dandori no yoki («bien organisé») ; dans la partie gauche kasegi («gain»), tsuie no naki («économe»), kannin zuyoki («persévérant»), kanai mutsumashiki («la paix du ménage») et yudan no naki («sans relâche»).

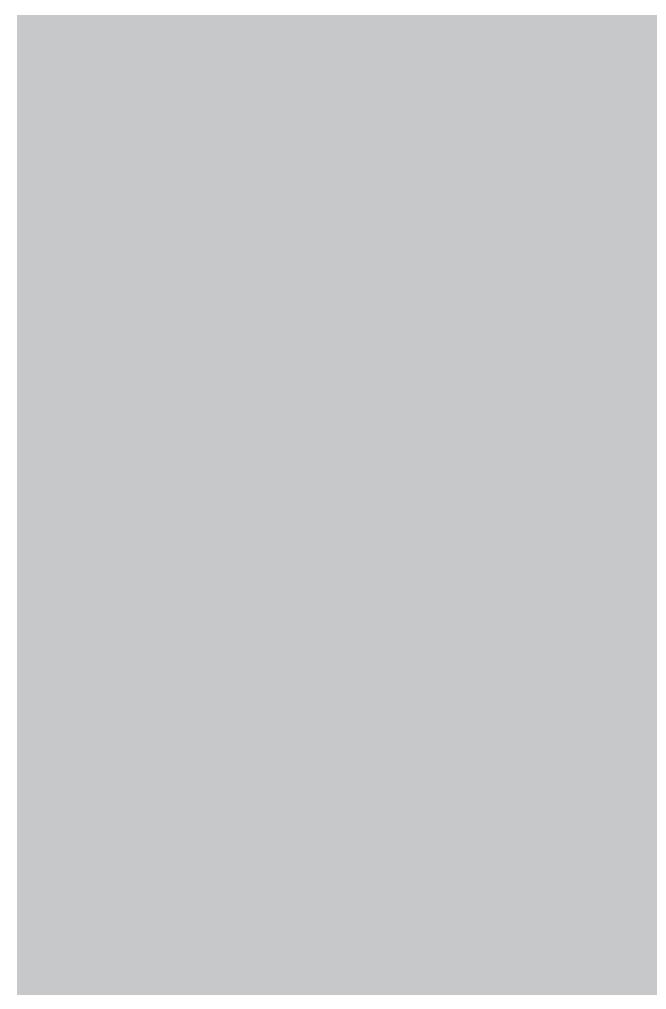

Figure 7. «Arbre aux feuilles d'or», Utagawa Yoshinobu, 18481860. D'après Inagaki, Edo no asobi-e, Tôkyô shoseki, 2000, p. 122 . 
Le tronc se lit, lui, de haut en bas: yorozu hodo no yoki («modéré»), jihi bukaki («charitable») et shôjiki («honnête»). On connaît aussi des arbres aux feuilles d'or invitant à la mollesse, d'autres transformés en cerisiers en fleurs abritant une joyeuse compagnie, en mélancoliques saules pleureurs, ou abritant de jeunes femmes élégantes ${ }^{24}$.

Quelques-uns fonctionnent même comme des calendriers, comme le montre une estampe de 1849 (Ka.ei 2, année du coq, cadet de la terre) (pl. 28, p. 111). Si l'apparence globale de l'arbre est inchangée, le texte qui s'y trouve dissimulé sert, lui, à indiquer l'alternance des mois - dont on se souvient qu'ils se disent fort opportunément tsuki. On lit, dans la partie droite, de haut en bas: shô dai, ni shô, san dai, shi shô no tsuki, go dai no tsuki, rokugatsu wa shô no tsuki ( $1^{\mathrm{er}}$ mois long, $2^{\mathrm{e}}$ court, $3^{\mathrm{e}}$ long, $4^{\mathrm{e}}$ court, $5^{\mathrm{e}}$ long, $6^{\mathrm{e}}$ court»). Dans la partie gauche, de haut en bas encore: shichi dai, hachi shô, ku dai, jû dai no tsuki, shimotsuki shô no tsuki, gokuzuki dai no tsuki ( $7^{\mathrm{e}}$ mois long, $8^{\mathrm{e}}$ court, $9^{\mathrm{e}}$ long, $10^{\mathrm{e}}$ long, $11^{\mathrm{e}}$ court, $12^{\mathrm{e}}$ long»). Le tronc se lit: shigatsu wa shô de urûzuki («Le mois intercalaire, court, est celui qui suit le $4^{\mathrm{e}} \gg$ ). Les invitations vertueuses ont ainsi été remplacées par la simple mention des mois, répartis en deux groupes de six branches et, pour le mois intercalaire, le plus problématique, dans le tronc. Le sac dans lequel se trouve l'arbre est en outre décoré du caractère qui désigne le coq dans le zodiaque (西). Le texte qui accompagne la figure explique que du sac de patience, dont les cordons ont laissé l'orifice entrouvert, s'échappent mille pièces d'or (Kannin no fukuro no kuchi no yurumi yori iza senkin no hana no harukaze). Un arbre aux feuilles d'or ne pousse habituellement pas dans un sac, mais dans un pot. Surtout, lorsqu'un «sac de patience» (kanninbukuro) se déchire, c'est pour laisser s'échapper la colère, celle qu'éprouve tout homme dont l'endurance a atteint ses limites ${ }^{25}$. Ici, généreusement ouvert, il laisse échapper un arbre calendaire.

Les arbres aux feuilles d'or sont peu nombreux dans notre corpus de calendriers en images. On comprend cependant que les artistes aient pu être séduits par les ressources de ce genre qui avait déjà fait la preuve de sa plasticité. Puisque les arbres pouvaient servir d'autres buts que l'exaltation au travail et à la vertu, pourquoi n'auraient-ils pu se faire aussi calendriers?

\section{Conclusion}

Des calendriers en images les plus simples aux plus complexes, l'analyse doit, on le voit, multiplier les outils et les références pour parvenir à une élucidation convaincante des codes, des sources et des métamorphoses repérés dans les images. Les quelques exemples présentés ici sont encore très loin d'épuiser l'ensemble du corpus, qui en compte plusieurs centaines. Ils illustrent toutefois plusieurs procédés importants qu'on y voit à l'œuvre, et peuvent 
constituer un point de départ pour une réflexion plus large. Plusieurs critères formels peuvent être dégagés, permettant de définir la façon dont les mois sont indiqués dans la figure, par exemple sous forme de motif figuratif ou de caractères écrits, dans la figure elle-même ou dans le texte qui l'accompagne. Les procédés repérés peuvent être présents simultanément dans une même image, ou faire appel à d'autres conventions en usage dans d'autres genres.

Le danger d'une étude de ce type est évidemment la simple élaboration d'une liste des règles et des exceptions à ces règles. L'exhaustivité étant ici d'emblée hors d'atteinte, toute typologie, même fine, ne peut que se révéler incomplète. Cette conclusion, pour décevante qu'elle soit, ne fait que prendre acte de la nature même des calendriers en images. Leurs écarts par rapport aux conventions font, en effet, eux aussi partie du code qu'ils mettent en place. La dissimulation, le détournement de moyens sont dans leur nature.

C'est là, peut-être, d'ailleurs la différence majeure entre les calendriers en images et les codes secrets habituels, auxquels leur fonctionnement peut d'abord faire penser. Les codes en effet reposent généralement sur un nombre fini de procédés, dépourvus de toute ambiguiité. Chaque indice y est doté d'un sens, et d'un seul, destiné à éliminer tout risque d'erreur dans le cryptage, comme dans le décryptage, du message à transmettre. La moindre incertitude pouvant être fatale, l'interprétation des indices obéit à des règles déterminées, auxquelles il est impossible de déroger sous peine de ne plus pouvoir accéder au message. On peut, par mesure de sécurité notamment, renouveler un code autant de fois que nécessaire. Chaque nouveau code n'en restera pas moins fermé, homogène et univoque. Les procédés à l'œuvre dans les calendriers en images sont, au contraire, flottants. Ils se rattachent à quelques grands principes, mais sont dans leur détail susceptibles d'aménagements multiples. Car ce n'est pas la lisibilité qui importe, mais le plaisir du jeu, c'est-à-dire, avant tout, de l'écart, cet écart que tous les codes cherchent justement à bannir, entre une image et ce qu'elle dissimule. Le secret, à vrai dire, n'importe guère non plus. Ne se bornent-ils pas à transmettre des informations connues de tous? Leur plus grand tour de force est peut-être de réussir des cryptages efficaces (la preuve en est qu'on peut les décrypter), à l'aide de règles incertaines, et variables. Un code ouvert, au service d'un secret de polichinelle, telle pourrait être une de leurs définitions. La frivolité a parfois des allures de défi. 


\section{NoTES}

1. À l'époque d'Edo (1603-1867), ces calendriers étaient le plus souvent appelés daishô-goyomi («calendriers [indiquant les mois] longs et courts»). Ils sont aujourd'hui désignés au Japon sous le terme egoyomi, que nous avons choisi d'adopter ici et que nous traduisons très littéralement par «calendriers (koyomi) en images $(e) »$.

2. Si la mode des calendriers en images ne dura que deux ans, leur production se poursuivit jusqu'à l'ère Meiji (1868-1912). Mais, imprimés de manière sporadique, ils se transformèrent en objets de curiosité et ne purent jamais retrouver leur première gloire.

3. Jusqu'en 1765 , et à l'exception de quelques ouvrages, des recueils poétiques en particulier, la plupart des livres et des estampes étaient gravés à l'aide d'une seule planche de trait, puis éventuellement agrémentés de couleurs au pinceau. Commandés dans un cadre privé, réalisés sur des papiers parfois très luxueux et à l'aide de pigments coûteux, les egoyomi favorisèrent la recherche de procédés d'impression en couleurs. Attribuée à Suzuki Harunobu (1725 ?-1770), la mise au point en 1765 de la polychromie en série à l'aide de plusieurs planches imprimées successivement sur une même feuille constitua un tournant dans l'histoire de l'estampe. Ces faits bien connus, ainsi que l'influence des techniques chinoises de polychromie, sont rappelés par exemple par Uchida Keiichi dans son Edo no shuppan jijô, Seigensha, 2007, p. 21-29.

4. Ils pratiquaient en particulier le kyôka, poème comique de 31 syllabes, très en vogue à partir des années 1755-1757.

5. Tels sont quelques-uns des procédés de cryptage les plus fréquents relevés par Matthi Forrer dans Egoyomi and Surimono, Their History and Development, J.C. Gieben, Uithoorn, 1979, p. 16. La fréquence de ces procédés est elle-même irrégulière. Le traitement des chiffres en motifs décoratifs est par exemple majoritaire dans les calendriers de l'ère Meiwa. Il reste utilisé, en concurrence avec l'alternance d'objets courts et longs et le moji-e, jusqu'en 1790 environ, moment à partir duquel on remarque la présence croissante de chiffres dans des poèmes.

6. On a en particulier puisé dans trois albums catalogués sous les titres suivants: Daishô goyomi harimaze-chô (un volume), Egoyomi harikomi-chô (un volume) et Egoyomi (deux volumes), tous accessibles aussi sur le site Internet de la Bibliothèque de la Diète (http://www.ndl.go.jp/jp/data/endl.html).

7. Il arrivait que le caractère 閏 fût remplacé par l'élément qui se trouve en son centre, 壬.

8. Soixante étant le plus petit commun multiple de dix et de douze, un cycle de soixante années constituait un ensemble considéré comme entier. Aujourd'hui encore, les Japonais célèbrent d'ailleurs avec une attention toute particulière leur soixantième anniversaire (kanreki).

9. Il était interdit par exemple, sous peine d'exil, même de copier des calendriers imprimés. Les egoyomi, produits de manière privée et non commercialisés, n'enfreignaient cependant pas l'interdiction. Ils n'avaient d'ailleurs pas vocation 
à remplacer les calendriers officiels, mais cherchaient simplement à en proposer une version différente pour un public d'amateurs, qui en faisaient un usage exclusivement privé.

10. Plus de cent egoyomi furent produits cette année-là, moins en 1766. On a avancé que l'année 1765 correspondait entre autres au 900 aniversaire de l'année où Sugawara no Michizane (845-903), patron des poètes, entra au service de l'empereur Seiwa (r. 858-876), et on a supposé que cette date aurait conduit à des concours entre groupes de poètes pour composer des calendriers en images. Mais aucun calendrier en images pour l'année 1765 ne semble présenter d'allusion particulière à Michizane. On a supposé aussi que la construction d'un observatoire en 1765 dans le quartier d'Ushigome, à Edo (aujourd'hui Tôkyô), avait pu susciter une curiosité particulière pour les calendriers. Cette hypothèse n'est toutefois étayée par aucune preuve. Les calendriers en images connurent un nouvel engouement entre 1785 et 1792 (Forrer, op. cit., p. 14). La substitution du calendrier grégorien au système luni-solaire, au cours de l'ère Meiji, les rendit tout à fait caducs.

11. Deux des albums de la Bibliothèque de la Diète, par exemple, proviennent des collections rassemblées par des spécialistes d'astronomie: Daishô goyomi harimaze-chô fut collationné par Ojima Sekiyû (1876-1948), et Egoyomi harikomi-chô par Shinjô Shinzô (1873-1938). Egoyomi provient, quant à lui, de la collection de Negishi Takeka (1839-1902). Aucun de ces personnages ne s'intéressait particulièrement à l'histoire de l'art, pas plus que les spécialistes auxquels on doit les ouvrages les plus importants sur le sujet. Sur les egoyomi dans l'histoire des calendriers, voir Okada Yoshirô, Nanbu egoyomi, Hôsei daigaku shuppankyoku, 1980, et Hasebe Kotondo, Daishô goyomi, Ryûkei shosha, 1988. L'ouvrage d'Okada, Edo no egoyomi, Taishûkan shoten, 2006, abondamment illustré et organisé de manière thématique, fournit une introduction commode au sujet. On pourra se reporter également à Yano Ken. ichi, Daishô goyomi wo yomitoku, Edo no kichi to yûmoa, Taishûkan shoten, Ajia bukkusu, $\mathrm{n}^{\circ}$ 25, 2000. En français, on consultera l'article de S. Niala, «Egoyomi», in Bulletin, Association franco-japonaise, $n^{\circ}$ 52, avril 1996, p. 32-39.

12. On renverra par exemple à Nishiki-e no tanjô, Edo shomin bunka no kaika (The Birth of Nichiki-e: Full-color Woodblock Prints and Edo Popular Culture), catalogue d'exposition, Musée Edo-Tôkyô, 1996. Les historiens d'art associent souvent les egoyomi aux surimono, ces estampes polychromes luxueuses, elles aussi produites et distribuées de manière privée, qui se caractérisent par la présence non pas d'indications calendaires (bien que certaines contiennent des mentions permettant d'identifier l'année pour laquelle elles furent imprimées), mais de poèmes. Les saitan surimono étaient même commandés spécialement pour le Nouvel An. Les ouvrages sur les surimono commencent souvent par une présentation rapide des egoyomi, mais ne s'y attardent pas. On le vérifiera dans l'introduction de Roger Keyes à son livre The Art of Surimono, Privately Published Japanese Woodblock Prints and Books in the Chester Beatty Library, Dublin, 1985, vol. 1, p. 13-40, ou encore dans l'article de Joan B. Mirviss, «A 
Hidden Legacy: The Surimono Collection of Franck Lloyd Wright», dans The Franck Lloyd Collection of Surimono, Weatherhill/Phoenix Art Museum, 1995, p. 11-35.

13. Okada présente ainsi un calendrier pour l'année 1770 représentant un marchand de sel, dans lequel l'artiste a mal localisé le mois intermédiaire. Voir Okada, Edo no egoyomi, p. 86.

14. Ce calendrier est commenté par Okada, op. cit., p. 42.

15. On pourra se reporter sur ce sujet à Nishiki-e no tanjô, Edo shomin bunka no kaika, en particulier les p. 66-67.

16. Claire-Akiko Brisset utilise le terme «cryptographie» à propos de l'un des ancêtres du moji-e, l'ashide (littéralement «écriture de roseau»). Voir par exemple: «Un cas de cryptographie religieuse et poétique dans le Japon ancien», Textuel $n^{\circ}$ 40: Écriture et typographie en Occident et en Extrême-Orient, Université Paris Diderot/CEEI, 2001, p. 29-44; "Art bouddhique et cryptographie dans le Japon du XII ${ }^{\mathrm{e}}$ siècle: le cas du Heike nôkyồ», dans Marianne Simon-Oikawa (dir.), Études japonaises nº 3: L'Écriture réinventée. Formes visuelles de l'écrit en Occident et en Extrême-Orient, Université Paris Diderot, GREJA/Les Indes savantes, Paris, 2007, p. 11-24.

17. Kanagaki Robun (1829-1894) fut un journaliste et écrivain, célèbre pour son humour.

18. Cette fête dite Shichigosan (littéralement «Sept, cinq, trois »), qui a lieu depuis 1873 le 15 novembre, est organisée pour les petites filles âgées de trois et sept ans et les petits garçons de cinq ans. Elle célèbre la croissance des enfants, qui se présentent avec leurs familles dans les sanctuaires et les temples, vêtus de leurs plus beaux habits.

19. Ce titre, que nous renonçons à traduire, repose sur une suite de jeux de mots à partir du titre d'un ouvrage de l'époque d'Edo intitulé Ono no Takamura uta jizukushi («Recueil de caractères mis en poèmes par Ono no Takamura»), compilation de poèmes composés sur des caractères d'écriture. Ono no Takamura (802-852), noble de cour de l'époque Heian célèbre pour son érudition, ne sert lui-même que de prête-nom à l'auteur de ce livre. Le titre de Hokusai, qui s'inscrit parmi une longue série de parodies de cet ouvrage, transforme le nom personnel Takamura en Bakamura (baka signifiant «idiot») - de sorte que la séquence Ono ga baka au début du titre peut se comprendre «je suis idiot»-, et le mot uta («poème») en muda («vain»). Voir à ce sujet Inagaki Shin.ichi, «Les jeux d'écriture à l'époque d'Edo», dans Claire-Akiko Brisset, Pascal Griolet, Chistophe Marquet et Marianne Simon-Oikawa (dir.), Du pinceau à la typographie, regards japonais sur l'écriture et le livre, École Française d'Extrême-Orient, «Études thématiques » n² 20, 2006, p. 231-259, en particulier notes 10 et 20 .

20. $C f$. Simon-Oikawa, «Écrire pour peindre: Les moji-e de Hokusai et de Hiroshige », Anne-Marie Christin et Atsushi Miura (dir.), Textuel ${ }^{\circ} 54$ : La Lettre et l'image: nouvelles approches, Université Paris Diderot/CEEI, 2007, p. 75-96.

21. $C f$. Simon-Oikawa, «Quelques exemples de moji-e dans deux recueils de petits 


\section{Marianne Simon-Oikawa}

métiers de l'époque d'Edo», in Japon pluriel 3, Arles, Philippe Picquier, 1999, p. 397-406. On lira aussi, en japonais, Chu Kyonkuk, «Iwayuru Moji-e tsukushi ni tsuite», dans Nobuhiro Shinji (dir.), Edo no bunji, Perikan-sha, 2004, p. 154-169.

22. Etchû est le nom d'une des régions administratives du Japon à l'époque d'Edo. Elle correspond à l'actuelle préfecture de Toyama.

23. Koyashi signifie littéralement «engrais», et même «fumier» ou «purin». En tombant sur le sol, la sueur enrichit naturellement la terre sur laquelle pousse l'arbre.

24. Pour des exemples d'arbres canoniques, et parodiques, on se reportera à Inagaki, Edo no asobi-e, p. 122-123.

25. L'expression kanninbukuro no o ga kireru signifie «être à bout de patience, se mettre en colère». 
Le temps codé: les calendriers en images (egoyomi) au Japon

\section{GLOSSAIRE}

Ansei (ère) 安政

asaoki 朝起き

ashide 葦手

baka 馬鹿

dai 大

dai no tsuki 大の月

Daikoku 大黒

Dainoshin 大之進

daishô no kai 大小会

daishô-goyomi 大小暦

Daishô goyomi harimaze-chô 大小暦張交帖

dandori no yoki 段取りの良き

$e($ image ») 絵

$e$ (《aîné ») 干

Ebisu 恵比寿

Edo 江戸

egoyomi 絵暦

Egoyomi (titre d'album) 恵合余見

Egoyomi harikomi-chô 絵暦貼込帳

Etchû (province) 越中

Gakutei Ryôsa (1786-1868) 岳亭梁左

gama no abura 蝦幕の油

hana-uri 花売り

hangontan 反魂丹

Heian 平安

hinoe 丙

hinoto 丁

isagi no yoki 潔の良き

jihi bukaki 慈悲深き

jikkan十干

jûnishi 十二支

Ka.ei (ère) 嘉永

kana 仮名

Kanagaki Robun (1829-1894) 仮名垣魯文

kanai mutsumashiki 家内むつましき

kane no naru $k i$ 金の成る木

kanji 漢字

kannin bukuro 堪忍袋

kanninbukuro no o ga kireru 堪忍袋の緒が切れる

kannin zuyoki 堪忍強き 


\section{Marianne Simon-Oikawa}

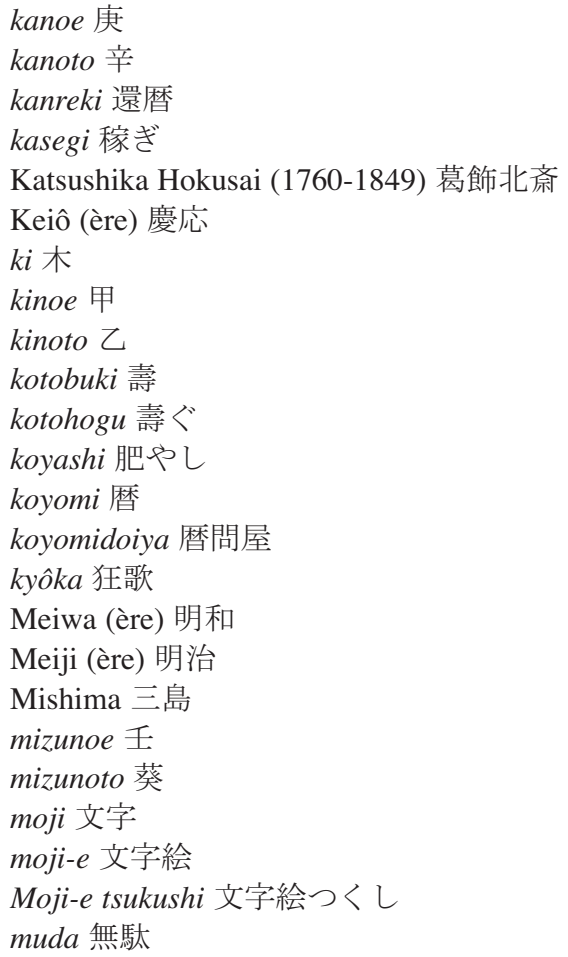


Le temps codé: les calendriers en images (egoyomi) au Japon

surimono 摺物

Suzuki Harunobu (1725?-1770) 鈴木春信

taihei 太平

Tenmei (ère) 天明

to 支

Tôkyô 東京

tora (signe du zodiaque) 寅

toridoshi 西年

Toyama (département) 富山

tsuchinoe 戊

tsuchinoto 己

tsuchinoto tori daishô 己西大小

tsuie no naki 費えの無き

tsuki 月

Tsukuba (Mont) 筑波山

$u r u ̂$ no tsuki 閏の月 ou 壬の月

Ushigome 牛込

$u t a$ 歌

Utagawa Yoshinobu (1838-1890) 歌川芳信

Yashima Gakutei (1786-1868) 八島岳亭

yôjô yoki 養生良き

yorozu hodo no yoki 萬程の良き

yudan no naki 油断の無き

yuzuriha 譲り葉 
